\title{
The Potential Detrimental Effects on Multiple Organ Systems in COVID-19 Patients
}

\author{
Zinat Mahal ${ }^{1 *}$ and Hasan M Zahid ${ }^{2}$ \\ ${ }^{1}$ Microbiology and Industrial Irradiation Division, Institute of Food and Radiation Biology, Bangladesh \\ ${ }^{2}$ Institute of Tissue Banking and Biomaterial Research, Bangladesh
}

*Corresponding author: Zinat Mahal, Microbiology and Industrial Irradiation Division, Institute of Food and Radiation Biology, AERE, Ganakbari, Savar, Dhaka-1349, Bangladesh.

To Cite This Article: Zinat Mahal, Hasan M Zahid, The Potential Detrimental Effects on Multiple Organ Systems in COVID-19 Patients. Am J Biomed Sci \& Res. 2021 - 11(5). AJBSR.MS.ID.001661. DOI: 10.34297/AJBSR.2021.11.001661.

Received: 眥 January 04, 2021; Published: 啙 January 20, 2021

\begin{abstract}
Severe acute respiratory syndrome coronavirus-2 (SARS-CoV-2) causes COVID-19, which spreads between people, mainly when an infected person stays in close contact with another one. The infection starts off with mild flu-like symptoms to severe in the several aspects. In the most cases, COVID-19 patients usually develop mild to moderate illness and recover at home stay with nominal medications. The usual signs of COVID-19 are fever, dry cough, loss of taste, tiredness etc., in very rare cases symptoms are difficulty in breathing, muscle pain, chest pain, loss of speech or movement etc. The deceased people from COVID-19 reveal the expected features of severe pneumonia or acute respiratory distress syndrome (ARDS). The virus seems to directly cause inflammation in the small capillaries or blood vessels leading to blood clots and damage heart, liver, brain and kidneys. In some people, it can affect the blood, digestive tract and immune system, causes multiple organ failure and resulting in death. Most critical patients showed signs of organ functions damage, including ARDS in 67\%, acute kidney injury (AKI) in 29\%, cardiac injury in 23\%, liver dysfunction in $29 \%$, and pneumothorax in $2 \%$. Since this virus causes serious health emergence in some extents, we should be more aware about COVID-19 disease. i.e., wearing mask, maintenance physical distancing, hand sanitization etc. In this mini review, we described the detrimental effects on the different organs in COVID-19 patients.
\end{abstract}

Keywords: SARS-CoV-2; COVID-19; Affected organs; Human body

\section{Introduction}

Coronavirus disease 2019 (COVID-19) is an infectious disease that is exposed from the severe acute respiratory syndrome coronavirus-2 (SARS-CoV-2), a novel coronavirus first detected in Wuhan, Hubei, China in December 2019 [1]. It bears single stranded enveloped positive sense RNA with four major structural proteins, e.g, spike (S), nucleocapsid (N), membrane (M), and envelope (E) protein [2] (Figure 1).

COVID-19 is closely related with SARS, and like most SARS they typically affect respiratory system in human. The infection starts with mild flu-like symptoms or no symptoms, and further progress to severe [3]. For the present global pandemic it is a burning time to understand the transmission and the effect of virus. This virus has been reported in almost 220 countries, areas or territories; $62,363,527$ cases have been confirmed with $1,456,687$ deaths as November 30, 2020 [4]. WHO is working in collaboration with different professionals including scientists to formulate effective vaccine(s). Currently more than 100 COVID-19 vaccine candidates are under development and human trial phase [5]. Current evidences suggest that COVID-19 may spread between people through direct, indirect (through contaminated objects or surfaces), or close contact with the infected person. They released from the mouth or nose when an infected person coughs, sneezes, speaks etc.

COVID-19 patients usually develop mild to moderate illness and recover without hospitalization. In the majority of the cases i.e. $80 \%$ exhibit mild symptoms, $14 \%$ have pneumonia, 5\% suffer 
from organ failure and in $2 \%$ cases show fatal [4,5]. The normal symptoms of COVID-19 are fever, dry cough, loss of taste or smell and tiredness. Severe symptoms are difficulty in breathing, muscle pains, chest pain, loss of speech or movement etc. [6]. People with mild symptoms who are otherwise healthy may manage their symptoms at home. But elder people without or with co-morbidities i.e., hypertension, diabetes, cardiac problems, respiratory issues and individuals on immune suppressing medications are at higher risk [7]. About 20\% of COVID-19 infected people require hospitalization to treat pneumonia, and many need assistance of oxygen. In about $5 \%$ of cases the pneumonia becomes so severe, patients need to admit in intensive care for breathing support [8]. Symptoms of COVID-19 infected patients are observed within 2-14 days after exposure [9].

\section{Fatal Effects on Different Organ Systems in COVID-19 Patients}

Deceased person from COVID-19 usually showed severe pneumonia or acute respiratory distress syndrome (ARDS). The virus causes inflammation in the small capillaries or blood vessels leading to blood clots and damage different organs. In some cases, it can affect blood, digestive tract and immune system, causes multiple organ failure and resulting in death. Most critical patients showed signs of organ functions damage, including ARDS in 67\%, acute kidney injury (AKI) in 29\%, cardiac injury in 23\%, liver dysfunction in $29 \%$, and pneumothorax in $2 \%$ [10]. The organs mostly affected in COVID-19 patients are (Figure 2):
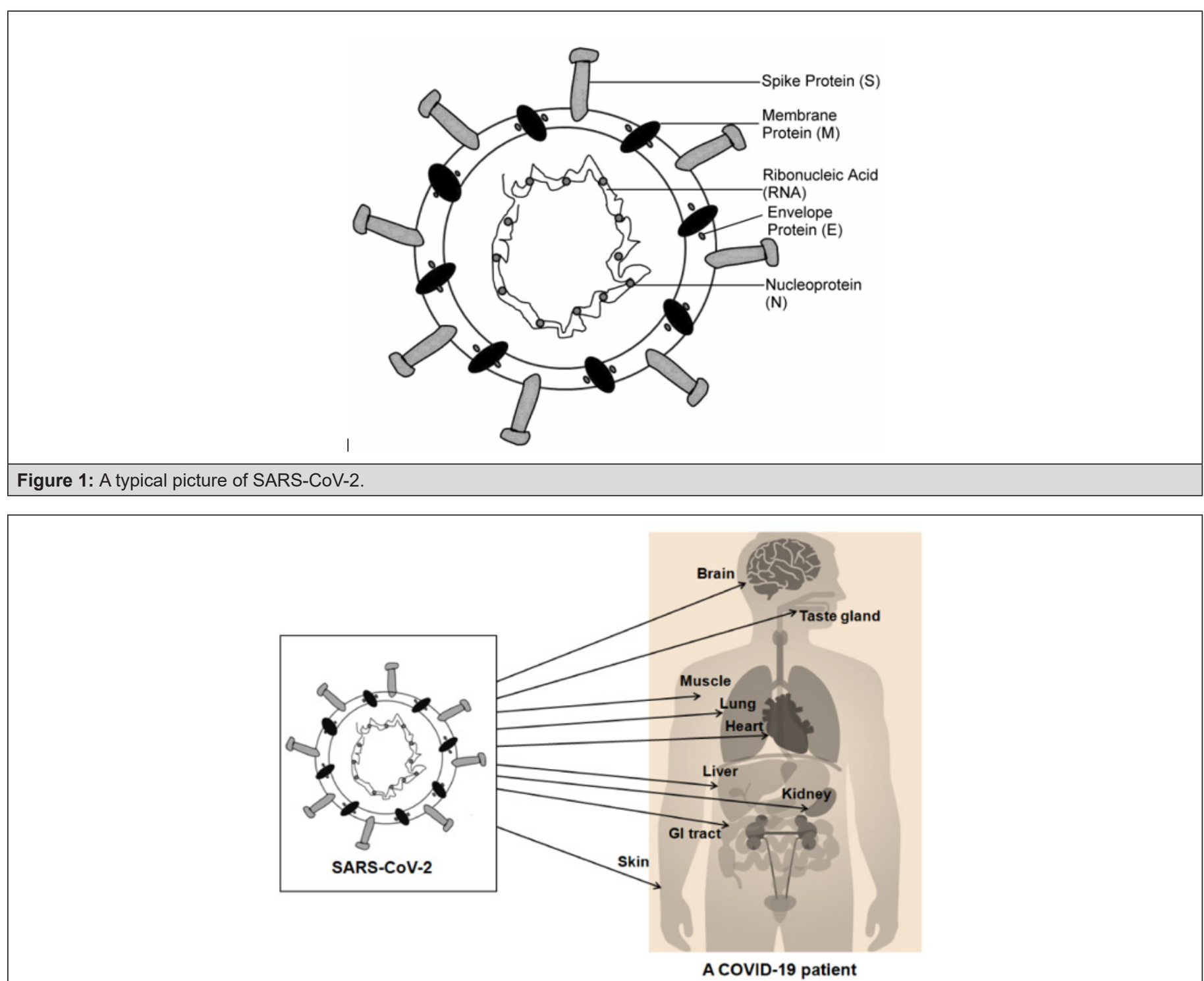

Figure: The affected organs in a COVID-19 patient.

Figure 2: The possible affected organs in COVID-19 patient. 


\section{Lung}

At the primary stage of an infection, the novel coronavirus can affect the respiratory tract. In lung, the alveolus is involved to transfer oxygen to the blood vessels. The blood vessels or capillaries carry oxygen to RBCs to deliver oxygen to all internal organs. After entering either through nose or mouth, the virus makes its way down into the alveoli and begins to cause injury to the respiratory system [11]. In alveoli, they use distinctive spike proteins $(S)$ to capture cells, then the viral proteins bust into cells through angiotensin converting enzyme 2 (ACE2) receptors which play role to control blood pressure and electrolytes. ACE2 receptors are present in the lungs, throat, gut, heart muscle, and kidneys [12]. In COVID-19, the wall and lining of the alveolus and capillaries are disrupted. The debris from the damage accumulates on the alveolus wall and thickens the lining, resulting the transfer of oxygen to RBC is impaired. Thus, a person often needs supplemental oxygen and very close monitoring in hospital [13].

\section{Skin}

In COVID-19 patients, skin appearances are also observed as rashes which can start at the onset of symptoms, but may also appear after a patient's hospitalization. The rashes look like widespread flat, pink scaly patches and raised spots. They may appear in clusters on all sides of the arms, legs, hands, and feet, or may be widespread over the body. Some patients also experience itchiness on the skin, develop hive-like lesions and widespread blisters. Skin rashes are related to vascular insufficiency in COVID-19 patients. This means the patients are not getting enough blood flow to the skin due to clots (thrombosis) in the skin's blood vessels. A patient having mild effect for COVID-19 infection shows perniosis-dusky or purple flat patches on the tips of the fingers or toes, moderately ill patient shows maculopapular rashes-flat and raised skin lesions and critically ill patient show acral or distal digital gangrene-dead tissue on the fingers or toes [14].

\section{Brain}

In Wuhan, China, $36 \%$ of 214 patients experienced neurologic complications. Sherry Chou reported that COVID-19 patients feel some disturbances in brain but enough is not known till now. Headache is another possible neurological symptom of COVID-19 listed by the CDC. Koralnik points to the possibility that a primary infection could take place in the brain stem. Injury to the brain and nervous system could also be a result of a cascade effect from loss of oxygen from damaged lungs that leads to multisystem organ failure [15]. COVID-19 is one of the most clot-causing diseases that can lead to life-threatening conditions i.e. strokes. Early data from China and Europe focused on this complication, and presently several hospitals in U.S. are starting to observe this incident [16]. Still, experts are unable to find the exact cause of strokes in coronavirus patients. A specific set of antibodies known as autoantibodies attack its own blood vessel cells, that may be partly responsible for the clotting risk associated with this disease [17].

\section{Taste Gland}

The olfactory cell types in the upper nasal cavity are most vulnerable to infection by SARS-CoV-2. However, the olfactory sensory neurons do not express the gene that encodes the ACE2 receptor, entry point of SARS-CoV-2 to human cells. Instead, ACE2 is expressed in cells that provide metabolic and structural support to olfactory sensory neuron. The findings suggest that the infection of non-neuronal cell types may be responsible for anosmia in COVID-19 patients. The virus changes the sense of smell in patients not by direct infections in neurons but by affecting the function of supporting cells. Datta et al. observed that ACE2 gene and transmembrane protease serine 2 (TMPRSS2) are widely distributed in cells of the human respiratory tract, those encode the main receptor protein by which COVID-19 enters into human cells. The analyses revealed that both ACE2 and TMPRSS2 are expressed by cells in the olfactory epithelium. They suggested that COVID-19 related anosmia may arise from a temporary loss of function of supporting cells in the olfactory epithelium, which indirectly causes changes to olfactory sensory neurons [18].

\section{Heart}

COVID-19 patients develop some heart problems including arrhythmias [19]. The virus may cause serious cardiac complications long after a patient has recovered from coronavirus. But the mechanism of heart damage is currently unclear. A report in the beginning of epidemic stated that the extent of cardiac injury among 41 patients hospitalized in Wuhan, China: 5-12\% had signs of cardiovascular damage [20]. They had elevated levels of cardiac troponin, a protein released in blood by the injured heart muscle. Some reports feature clinical scenarios in which patients initial symptoms were cardiovascular rather than respiratory distress. Although experts don't yet have conclusive data on the percentage of patients who have heart damage for COVID-19 infections, estimates around 25-30\% [21]. People with preexisting heart disease are at a greater risk for severe cardiovascular and respiratory complications by this virus. In people with existing heart-vessel blockages, infection, fever and inflammation can destabilize previously asymptomatic fatty plaques inside the heart vessels. Some people may experience heart damage that mimics heart attack injury even if their arteries lack the fatty, calcified flowlimiting blockages known to cause classic heart attacks. Fever and inflammation accelerate heart rate and increase metabolic demands on many organs including heart. The stresses are compounded if the lungs are infected and incapable of exchanging oxygen and carbon dioxide optimally. This impaired gas exchange can further diminish oxygen supply to the heart muscle. 


\section{Kidney}

The kidney damage is presented by AKI, mild proteinuria, hematuria, or slight elevation of blood urea nitrogen (BUN) and creatinine. The presence of higher concentrations of virus may lead to renal damage. Clinical studies observed renal failure in $50 \%$ patients in ICU, and around 5-10\% patients for dialysis [22]. AKI is more common among patients with more severe diseases, particularly in those recovering in the ICU [23]. The people are mostly at risk for AKI with COVID-19 infection are people with diabetes, high blood pressure and chronic kidney disease [24]. Detection of coronavirus in the kidneys and urine of patients with SARS-CoV and SARS-CoV-2 supports the theory that the virus can directly damage kidneys [25]. In one autopsy in kidney transplant patients who died of COVID-19, viral inclusion structures were found in the endothelial cells of the kidney [26]. Viral infection could induce tubular damage through the deposition of MAC complex on tubules and infiltration of CD68+ macrophages in the tubule interstitium. The virus can also force its way into the kidney by binding directly to kidney receptors [27]. In the most extreme cases, patients who go into acute renal failure lose their ability to remove waste and balance fluids in the body, and need dialysis or mechanical purification of their blood.

\section{Muscle}

Musculoskeletal symptoms may develop during coronavirus infections. Joint and muscle pains are described in $<10 \%$ of cases, whereas anthralgia is reported in 15\% of COVID-19 patients but myalgia is more frequent (44\%). Patients of COVID-19 may get pain in arms, legs, or back that develops spontaneously with no injury. Typically, in a coronavirus infection, the pain is in muscles rather than in joints. But if someone has an arthritic joint in his arm or leg, the virus may exaggerate the symptoms. The pain may be severe and limiting. While the coronavirus can cause permanent damage to important organs, such as the lungs and kidneys, it is not known to cause any permanent damage to bones and joints. Systemic inflammation may play a role in bone and joint tissue physiology in patients with COVID-19 [28].

\section{Liver}

In the first COVID-19 confirmed case in U.S., there was an increased level of hepatic enzymes during the treatment, which suggested that SARS-CoV-2 infection can directly affect liver [29]. The mechanism of liver injury is unknown but the expression of ACE2 receptor was found abundant in the endothelial cells of liver and bile duct cells [30]. The infection of this virus can also lead to liver injury with the elevated liver enzymes. The incidence of liver injury ranges from 15-53\% [31]. Severe COVID-19 patients have higher levels of aspartate aminotransferase (AST), alanine aminotransferase (ALT), total bilirubin (TBL), gammaglutamyl transferase, alkaline phosphatase, low albumin level, and prolonged prothrombin time than non-severe patients [32]. It is assumed that direct viral infection of hepatocytes, immunemediated inflammation, i.e. cytokine storm and drug hepatotoxicity may contribute to liver injury in critically ill patients [33]. Autopsy and biopsy report can also reveal liver injury associated with SARSCoV-2 infection [34].

\section{Gastrointestinal (GI) Tract}

SARS-CoV-2 affects GI tract, 60 studies with 4243 patients from China, Singapore, South Korea, United Kingdom, and United States showed a pooled prevalence of GI symptoms (17.6\%). Another pooled data from 11 articles showed that 2645 COVID-19 patients from China, Hong Kong and Singapore developed different GI symptoms. GI symptoms varied and usually preceded pulmonary symptoms by 1-2 days. These include anorexia (26.8\%), nausea and vomiting (10.2\%), diarrhea (12.5\%), and abdominal pain (9.2\%) [33]. Digestive symptoms were also reported among 204 COVID-19 patients in a descriptive, cross sectional, multicenter study during the initial outbreak in Wuhan, China. In that study, $41.6 \%$ of COVID-19 patients suffered nausea or vomiting, and $17.2 \%$ of COVID-19 patients suffered from diarrhea [34]. Notably, patients with severe disease and intensive care unit (ICU) patients were found with a higher incidence of diarrhea, nausea, vomiting, abdominal pain, and anorexia than those with non severe disease and non ICU patients [35]. GI epithelial and liver cells express ACE2, the major receptor of SARS-CoV-2. It is reported that ACE2 receptor expression is approximately 100 -fold higher in the GI tract than in the respiratory system [36]. So, it is understandable that like other ACE2 expressing organs digestive system are also vulnerable to COVID-19 attack.

\section{Super Infection}

ACE2 is widely distributed in cells of the human respiratory tract, which encodes the main receptor protein that SARS-CoV-2 targets to gain entry into human cells. TMPRSS2 encodes an enzyme also thought to be important for SARS-CoV-2 entry into the cell. The immune system works hard to fight the invader in COVID-19 patients. The resultant inflammation due to the defense response set off a cascade of immune signals that affect various organs. As a result, the body becomes more vulnerable to infection with another bacterium or virus on top of the COVID-19, as a super infection.

\section{Conclusion}

SARS-CoV-2 virus is showing its devastating effect in the world for about one year. So, now-a-days it's a burning issue to make the possible solutions of potential harmful effects on the different organs in our body. Even if the virus affects most of the important $\operatorname{organ}(\mathrm{s})$ in the human body; fortunately, they are not the death sentence for most people in the world. Factors associated with the severity of this disease include male sex, advanced age, and the presence of comorbidities including hypertension, diabetes 
mellitus, cardiovascular diseases, asthma, chronic kidney disease etc. Immuno-compromised patients are also vulnerable to this disease. We all should maintain healthy practices such as awareness of mass people, hand sanitization, wearing mask, maintenance of social distancing etc. to restrict the pandemic and return life to normal soon.

\section{Conflict of Interest}

The authors declare no compete of interest.

\section{References}

1. Wu P, Hao X, Lau EHY, Wong JY, Leung KSM, et al. (2020) Real-time tentative assessment of the epidemiological characteristics of novel coronavirus infections in Wuhan, China. Euro Surveill 25(3): 2000044.

2. Naqvia AAT, Fatimab K, Mohammada T, Fatimac U, Singhd IK, et al. (2020) Insights into SARS-CoV-2 genome, structure, evolution, pathogenesis and therapies: structural genomics approach. Biochem et Biophysic Acta (BBA)-Mol Bas Dis 1866(10): 165878.

3. Petrosillo N, Viceconte G, Ergonul O, Ippolito G, Petersen E (2020) COVID-19, SARS and MERS: are they closely related? Clin Microbiol Infect 26(6): 729-734.

4. https://www.who.int/emergencies/diseases/novel-coronavirus-2019

5. https://www.who.int/emergencies/diseases/novel-coronavirus-2019/ covid-19-vaccines

6. World Health Organization (2020) Report of the WHO-China joint mission on coronavirus disease 2019 (COVID-19). WHO, Geneva, Switzerland.

7. Madjid M, Safavi-Naeini P, Solomon SD, Vardeny O (2020) Potential effects of coronaviruses on the cardiovascular system: a review. JAMA Cardiol 5(7): 831-840.

8. Wark PP (2020) Here's what we know so far about the long-term symptoms of COVID-19. The Convers.

9. World Health Organization (2020) Report of the WHO-China Joint Mission on coronavirus disease 2019 (COVID-19). WHO, Geneva, Switzerland.

10. Masoud Z, Dyal NP (2020) Characteristics of SARS-CoV-2 infection, potential therapies for COVID-19.

11. Guite H (2020) Fact checked by Catherine Carver, MPH, Newsletter, Medical News.

12. Sriram K, Insel PA (2020) A hypothesis for pathobiology and treatment of COVID-19: The centrality of ACE1/ACE2 imbalance. Br J Pharmacol 177(21): 4825-4844.

13. Wichman D, Jan-Peter S, Lütgehetmann M, Steurer S, Edler C (2020) Autopsy findings and venous thromboembolism in patients with COVID-19. Ann Intern Med 6(2020): M20-2003.

14. Maderal A (2020) How does COVID-19 affect the skin? UMiami Health News.

15. Nania R (2020) This is what the coronavirus can do to your brain.

16. Dangerous blood clots increasingly found in COVID-19 patients by Associated Press, Health. (2020).
17.Zuo Y, Estes SK, Ali RA, Gandhi AA, Yalavarthi S, et al. (2020) Prothrombotic autoantibodies in serum from patients hospitalized with COVID-19. Sci Transl Med 12(570): eabd3876.

18. Jiang k (2020) How COVID-19 causes loss of smell, News \& Research.

19. Farrokhran SB, Rasekhi RT, Gill D, Babapoor S, Amanullah A (2020) Arrhythmia in COVID-19, SN Comprehen. Clin Med 14: 1-6.

20. Pesheva E (2020) Coronavirus and the heart.

21. Weinstock P (2020) COVID-19's toll on the heart, AARP.

22. Henderson E (2020) First extensive review of COVID-19's effects on all affected organs outside the lungs.

23. Durvasula R, Wellington T, Namara EM, Watnick S (2020) COVID-19 and kidney failure in the acute care setting: our experience from Seattle. Am J Kid Dis 76(1): 4-6.

24. Weinstock CP (2020) COVID-19 causing kidney damage, dialysis challenges for patients AARP.

25. Cheng Y, Luo R, Wang K, Zhang M, Wang Z, et al. (2020) Kidney disease is associated with in-hospital death of patients with COVID-19. Kid Int 97(5): 829-838.

26. Varga Z, Flammer AJ, Steiger P, Haberecker M, Andermatt R, et al. (2020) Endothelial cell infection and endotheliitis in COVID-19. Lancet 395(10234): 1417-1418.

27. Diao B, Wang C, Wang R, Feng Z, Tan Y, et al. (2020) Human kidney is a target for novel severe acute respiratory syndrome coronavirus-2 (SARS-CoV-2) infection. medRxiv.

28. Schett G, Manger B, Simon D, Caporali R (2020) COVID-19 revisiting inflammatory pathways of arthritis. Nat Rev Rheumat 16(8): 465-470.

29. Holshue L, DeBolt C, Lindquist S, Lofy KH, Wiesman J, et al. (2020) First case of 2019 novel coronavirus in US. N Engl J Med 382(10): 929-936.

30. Zhang C, Shi L, Wang FS (2020) Liver injury in COVID-19: Management and challenges. Lancet Gastroenterol Hepatol 5(5): 428-430.

31. Clinical insights for hepatology and liver transplant providers during the COVID-19 pandemic, AASLD.

32. Aguila EJT, Cua IHY, Dumagpi JEL, Francisco CPD, Raymundo NTV, et al. (2020) COVID-19 and its effects on the digestive system and endoscopy practice. JGH 4(3): 324-331.

33. Lei P, Mi M, Pengcheng Y, Sun Y, Wang R, et al. (2020) Clinical characteristics of COVID-19 patients with digestive symptoms in Hubei, China: A descriptive, cross-sectional, multi-center study. Am J Gastroenterol 115(5): 766-773.

34. Guan WJ, Ni ZY, Hu Y (2020) Clinical characteristics of coronavirus disease 2019 in China. N Engl J Med 382: 1708-1720.

35. Zhang H, Kang Z, Gong H, Xu D, Wang J, et al. (2020) Digestive system is a potential route of COVID-19: an analysis of single-cell co-expression pattern of key proteins in viral entry process. Gut 69(6): 1010-1018.

36. Gheblawi M, Wang K, Viveiros A, Nguyen Q, Zhong JC, et al. (2020) Angiotensin-converting enzyme 2: SARS-CoV-2 receptor and regulator of the renin-angiotensin system celebrating the $20^{\text {th }}$ anniversary of the discovery of ACE2. Circ Res 126(10): 1456-1474. 